\title{
Stirling Thermodynamics using Phasor Notation
}

\author{
David M. Berchowitz ${ }^{*}$ \\ Stirling Ultracold, 6000 Poston Rd., Athens, OH 45701, USA
}

\begin{abstract}
Phasor mathematics is used to develop the isothermal Stirling cycle and extended to the ideal adiabatic Stirling cycle. The results are developed for piston - piston (alpha) machines and displacer - piston (beta and gamma) machines. The effect of non-ideal regeneration is handled by defining a regenerator effectiveness ratio. The importance of the amplitude pressure ratio (pressure amplitude to the mean pressure) is developed and shown to be a useful parameter when evaluating the effect of dead volume or when applying simple cycle analyses. The analysis is developed for both power producing and cooling engines. The utility of these analyses is discussed with respect to calibrated results of real machines.
\end{abstract}

\section{Background}

Much like the Otto and Diesel cycles are the classic cycles used to describe the spark and compression ignition engines, the cycle described by Schmidt in 1871[1] has become the classic Stirling cycle. This is more about the Schmidt analysis being mathematically tractable rather than its reflection of the real cycle. The major assumption of Schmidt's analysis is that the gas in the working spaces and attendant heat exchangers is at their respective constant upper and lower cycle temperatures, and that regeneration is perfect. The gas is therefore isothermal everywhere but with spatial temperature distribution as indicated in Fig. 1. The assumption of isothermal working spaces is the most limiting aspect of the analysis. Schmidt also assumed that the volumes of the working spaces vary sinusoidally as is shown in a diagram from his original analysis of Lehmann's machine (Fig. 2).

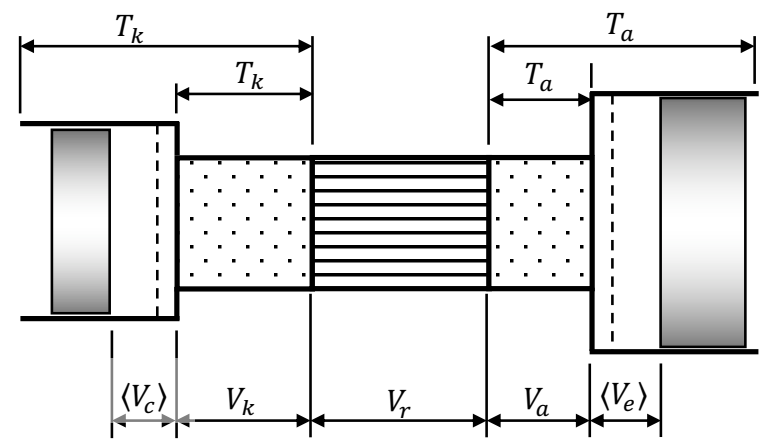

Isothermal Assumption: Working spaces are at constant adjacent heat exchanger temperature

Adiabatic Assumption: Working spaces are adiabatic and heat exchangers isothermal

Fig. 1. Isothermal and adiabatic assumptions

\footnotetext{
* dberchowitz@globalcooling.com
} 


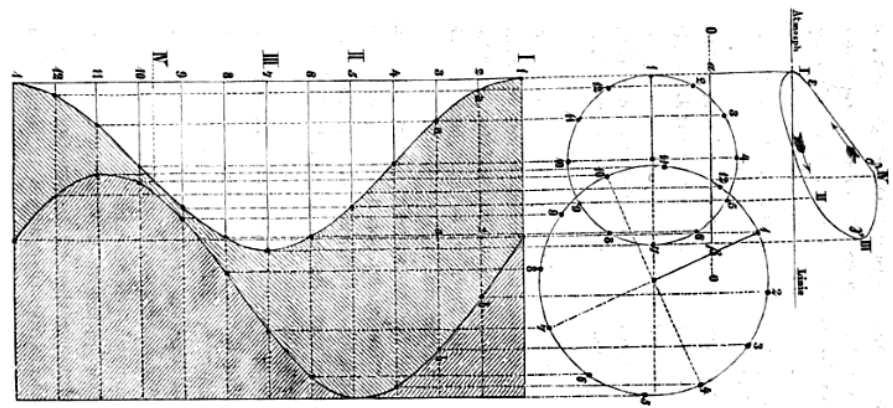

Fig. 2. Sinusoidal volume variations from Schmidt's original paper

In what follows, an ideal analysis will be developed using phasor notation that includes the effect of regeneration and adiabatic working spaces. In all other respects, the general assumptions are identical to those used by Schmidt.

\section{Analysis}

Following the method developed by Chen et al [13], we start by assuming that the following varying parameters are represented by first harmonics.:

$$
\begin{array}{ll}
V=\langle V\rangle\left(1+\delta V \mathrm{e}^{j(\alpha+\theta)}\right)=\langle V\rangle\left(1+\delta \widehat{V} \mathrm{e}^{j \theta}\right) & \text { working space volumes } \\
T=\langle T\rangle\left(1+\delta T \mathrm{e}^{j(\zeta+\theta)}\right)=\langle T\rangle\left(1+\delta \widehat{T} \mathrm{e}^{j \theta}\right) & \text { working space temperatures } \\
m=\langle m\rangle\left(1+\delta m \mathrm{e}^{j(\varphi+\theta)}\right)=\langle m\rangle\left(1+\delta \hat{m} \mathrm{e}^{j \theta}\right) & \text { working space masses }
\end{array}
$$

Where subscripts $c$ and $e$ will indicate compression and expansion spaces respectively, $\delta$ indicates the ratio of amplitude to mean value $\langle x\rangle$.

Following the Schmidt analysis, the total mass of gas in the engine is given by the sum of the gas masses in the various spaces

$$
m_{t}=\frac{p}{R}\left(\sum_{i=c, e} \frac{V_{i}}{T_{i}}+\sum_{i=k, r, a} \frac{V_{i}}{T_{i}}\right)
$$

Substituting for the varying volumes and temperatures, (1) and (2), and solving for the pressure gives:

$$
p=\frac{m_{t} R}{\sum_{i=k, r, a} \frac{V_{i}}{T_{i}}+\sum_{i=c, e} \frac{\left\langle V_{i}\right\rangle\left(1+\delta \widehat{V}_{i} \mathrm{e}^{\mathrm{j} \theta}\right)}{\left\langle T_{i}\right\rangle\left(1+\delta \widehat{T}_{i} \mathrm{e}^{\mathrm{j} \theta}\right)}}
$$

Noting that $\delta T_{i}<<1$, we use the Binomial theorem and rewrite as follows

$$
p \approx \frac{m_{t} R}{\sum_{i=k, r, a} \frac{V_{i}}{T_{i}}+\sum_{i=c, e} \frac{\left\langle V_{i}\right\rangle}{\left\langle T_{i}\right\rangle}\left(1+\delta \widehat{V}_{i} \mathrm{e}^{\mathrm{j} \theta}\right)\left(1-\delta \widehat{T}_{i} \mathrm{e}^{\mathrm{j} \theta}\right)}
$$

And, ignoring second order small terms 
$p \approx \frac{m_{t} R}{S} \frac{1}{1-\frac{1}{S} \sum_{i=c, e} \frac{\left\langle V_{i}\right\rangle}{\left\langle T_{i}\right\rangle}\left(\delta \widehat{T}_{i}-\delta \widehat{V}_{i}\right) \mathrm{e}^{\mathrm{j} \theta}}=\frac{m_{t} R}{S}\left(\frac{1}{1-\delta p \mathrm{e}^{\mathrm{j}(\beta+\theta)}}\right)$

where $S=\sum_{i=c, e} \frac{\left\langle V_{i}\right\rangle}{\left\langle T_{i}\right\rangle}+\sum_{i=k, r, a} \frac{V_{i}}{T_{i}}$ and $\delta p$ is the pressure amplitude ratio.

We now need a result that relates $m_{t}$ to the mean pressure $\langle p\rangle$. This is done by defining the mean cyclic pressure (also referred to as the charge pressure) as follows:

$\langle p\rangle=\frac{1}{2 \pi} \oint \frac{m_{t} R}{S}\left(\frac{1}{1-\delta p \mathbf{R}\left(\mathrm{e}^{\mathrm{j}(\beta+\theta)}\right)}\right) \mathrm{d} \theta$

Which, from standard integral tables [9] gives:

$\langle p\rangle=\frac{m_{t} R}{S} \frac{1}{\sqrt{1-\delta p^{2}}}$

Substituting into (5), the pressure may now be written:

$p=\langle p\rangle \frac{\sqrt{1-\delta p^{2}}}{1-\delta \hat{p} \mathrm{e}^{\mathrm{j} \theta}} \quad$ where $\quad \delta \hat{p}=\frac{1}{S} \sum_{i=c, e} \frac{\left\langle V_{i}\right\rangle}{\left\langle T_{i}\right\rangle}\left(\delta \widehat{T}_{i}-\delta \widehat{V}_{l}\right)$

This has the same form as the Schmidt isothermal result except that the $\delta T$ s would be zero.

Expanding (7) by Fourier series, gives the following result:

$p=\langle p\rangle\left[1+\frac{2}{\delta p}\left(1-\sqrt{1-\delta p^{2}}\right) \cos (\beta+\theta)+\frac{1}{2}\left(\sqrt{1-\delta p^{2}} \delta p^{2}\right) \cos 2(\beta+\theta)\right] \ldots$

$p=\langle p\rangle\left[1+\delta p_{1} \cos (\beta+\theta)+\delta p_{2} \cos 2(\beta+\theta)\right] \ldots$

The amplitude of the first harmonic is therefore given by:

$\delta p_{1}=\frac{2}{\delta p}\left(1-\sqrt{1-\delta p^{2}}\right)$ and has a phase of $\beta$.

For beta or gamma configurations (Fig. 3), it is convenient to express the volume variations in terms of the piston and displacer motions relative to the piston.

$$
\begin{aligned}
& V_{c}=\left\langle V_{c}\right\rangle+\left[\left(A_{d}-A_{R}\right) X_{d} \angle \phi-A_{p} X_{p}\right] \mathrm{e}^{\mathrm{j} \theta}=\left\langle V_{c}\right\rangle+\left|V_{c}\right| \angle \alpha_{c} \mathrm{e}^{\mathrm{j} \theta}=\left\langle V_{c}\right\rangle\left(1+\delta \widehat{V}_{c} \mathrm{e}^{\mathrm{j} \theta}\right) \\
& V_{e}=\left\langle V_{e}\right\rangle-A_{d} X_{d} \angle \phi \mathrm{e}^{\mathrm{j} \theta}=\left\langle V_{e}\right\rangle+\left|V_{e}\right| \angle \alpha_{e} \mathrm{e}^{\mathrm{j} \theta}=\left\langle V_{e}\right\rangle\left(1+\delta \widehat{V}_{e} \mathrm{e}^{\mathrm{j} \theta}\right)
\end{aligned}
$$

The volume, motion and pressure phasors are shown in Fig. 4. For cooling engines, the pressure leads piston motions and for power engines, the pressure lags piston motions. This is explained in [12]. The presence of the displacer rod results in the total volume variation not being slightly off anti-phase to the piston motions.

Taking $V_{c}$ as the reference phasor, a phasor diagram for the pressures and volumes would look as in Fig. 5. When the total volume is maximum, the pressure would be close to minimum (depending on temperatures). We will proceed further assuming $V_{c}$ as reference phasor. 


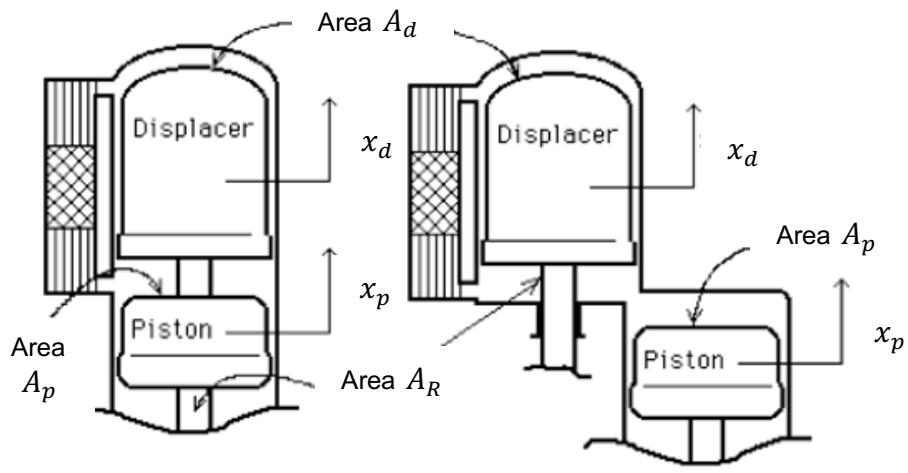

(a) Beta configuration

(b) Gamma configuration

Fig. 3. Piston and displacer motions for beta and gamma configurations

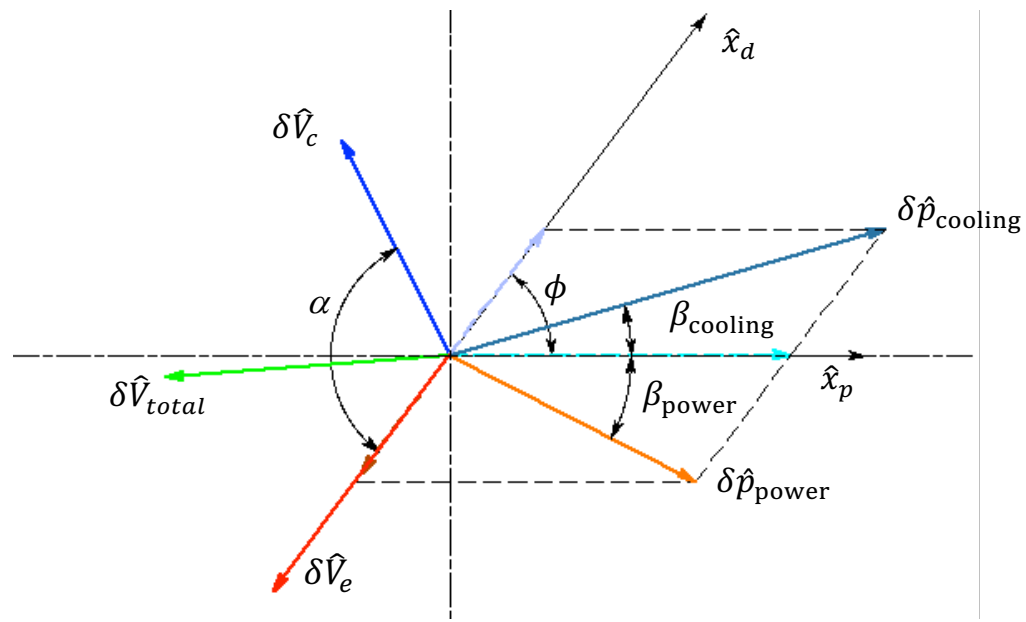

Fig. 4. Relative phase relationships of displacements, volumes and pressure with respect to $\hat{x}_{p}$ [12]

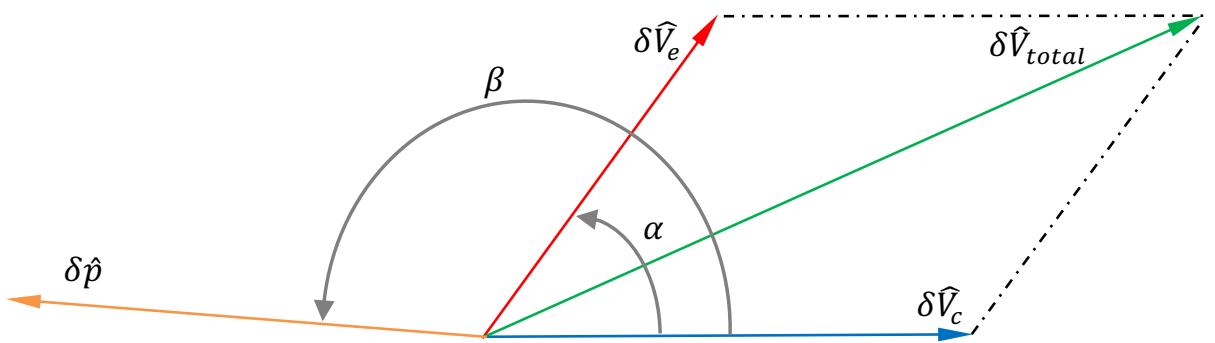

Fig. 5. Phasor representation of working space volumes and pressure with respect to $\widehat{V}_{c}$

For the adiabatic case, $T_{c}$ and $T_{e}$ are time dependent. The procedure for finding these expressions is outlined in Appendix A.

By inspection of a simple control volume containing either the rejector or acceptor, there is no net enthalpy transferred across the heat exchanger / regenerator interface. The heat transfer must therefore equal the work in the associated working space:

$W=Q=\oint p \mathrm{~d} V$ 
Substitution of (7) and the derivative of $V$ from (1) into the above, yields:

$$
W=Q=-\langle p\rangle \sqrt{1-\delta p^{2}}\langle V\rangle \delta V \oint \frac{\sin (\alpha+\theta)}{1-\delta p \mathbf{R}\left(\mathrm{e}^{\mathrm{j}(\beta+\theta)}\right)} \mathrm{d} \theta
$$

The solution of (13) involves a certain amount of manipulation and has been solved many times in the literature; in approximate form [2], and exact [3, 4] and [5]. Here, the final exact solution is given:

$$
W=Q=2 \pi\langle V\rangle\langle p\rangle \frac{\delta V}{\delta p}\left(\sqrt{1-\delta p^{2}}-1\right) \sin (\alpha-\beta)=-\pi\langle V\rangle\langle p\rangle \delta V \delta p_{1} \sin (\alpha-\beta)
$$

Each working space is therefore:

$$
\begin{aligned}
& W_{c}=Q_{k}=2 \pi\left\langle V_{c}\right\rangle\langle p\rangle \delta V_{c} \delta p_{1} \sin (-\beta) \quad(\alpha \text { is zero here }) \\
& W_{e}=Q_{a}=2 \pi\left\langle V_{e}\right\rangle\langle p\rangle \delta V_{e} \delta p_{1} \sin (\alpha-\beta)
\end{aligned}
$$

The solution to obtain the pressure is iterative and proceeds as follows:

1) Assume initial values of $\delta T_{c}$ and $\delta T_{e}$ equal to zero.

2) Solve for the pressure (7). On first pass, this will be the Schmidt result

3) Solve for the mass flow amplitudes and the flow reversal points, $\varphi$ (A.20).

4) Solve for the constants $B_{1}$ and $C_{2}$ for each space (A.28) and (A.31). $C_{2}$ is almost equal to $B_{1}$ and can be assumed so for most situations.

5) The temperature terms, $\langle T\rangle$ and $\delta T$ can now be found from (A.23) and (A.24) after evaluating the various $B$ constants from (A.14) and (A.18).

6) Iterate on the mean temperatures $\left\langle T_{c}\right\rangle$ and $\left\langle T_{e}\right\rangle$ back to (2) until desired tolerance is obtained. Convergence of better than $1 \%$ on the mean temperatures is usually obtained in less than eight iterations.

7) Finally the cyclic works $W_{c}$ and $W_{e}$ are obtained from Equations (15) and (16).

8) Harmonics of the pressure may be obtained from the Fourier series (8).

Pressure, temperature and working space mass flow profiles are shown in Figs. 6, 7 and 8 for a representative machine. Fig. 9 shows the phasor diagram of these quantities.

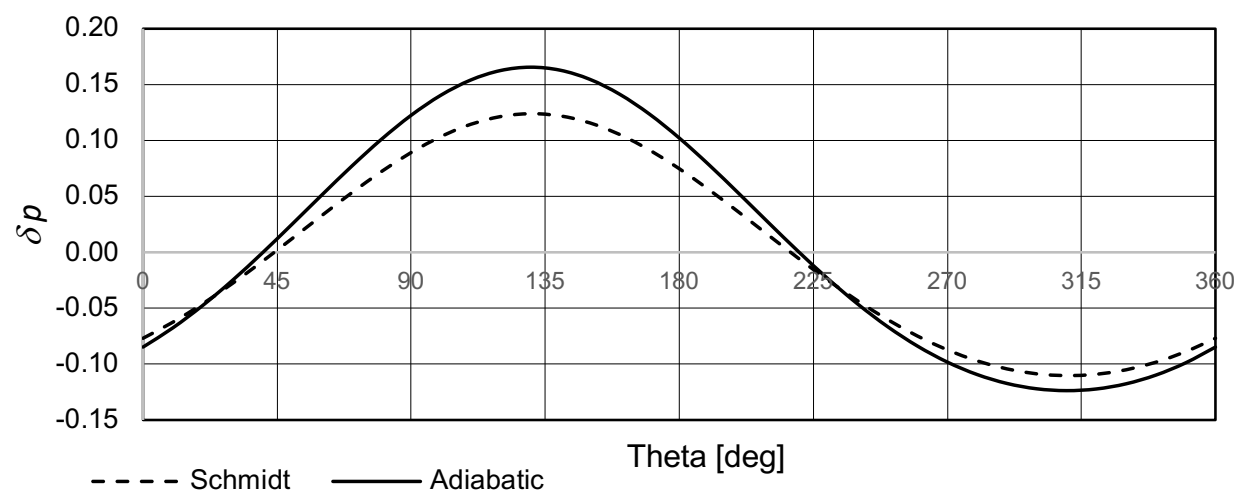

Fig. 6. Pressure Profiles for Adiabatic and Schmidt 


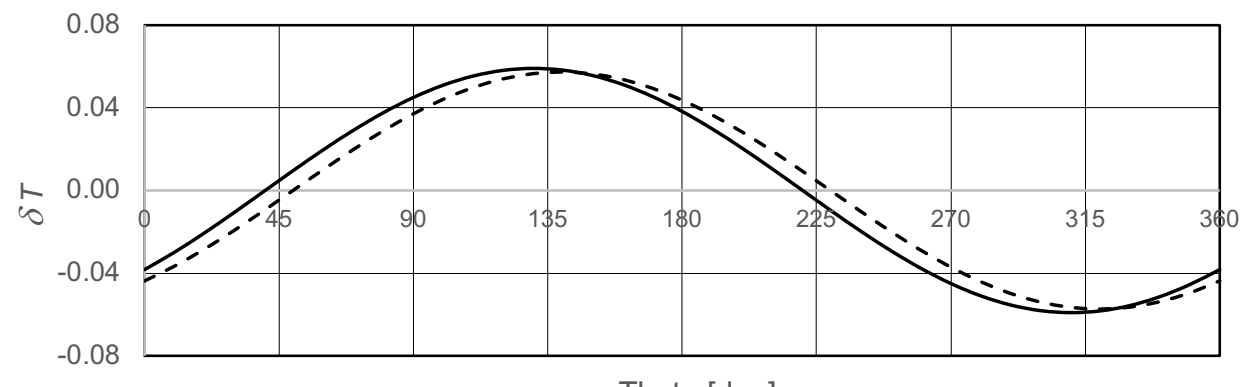

Theta [deg]

Temperature Ratio compression space - - - Temperature Ratio expansion space

Fig. 7. Temperature profiles (from Equation A.24)

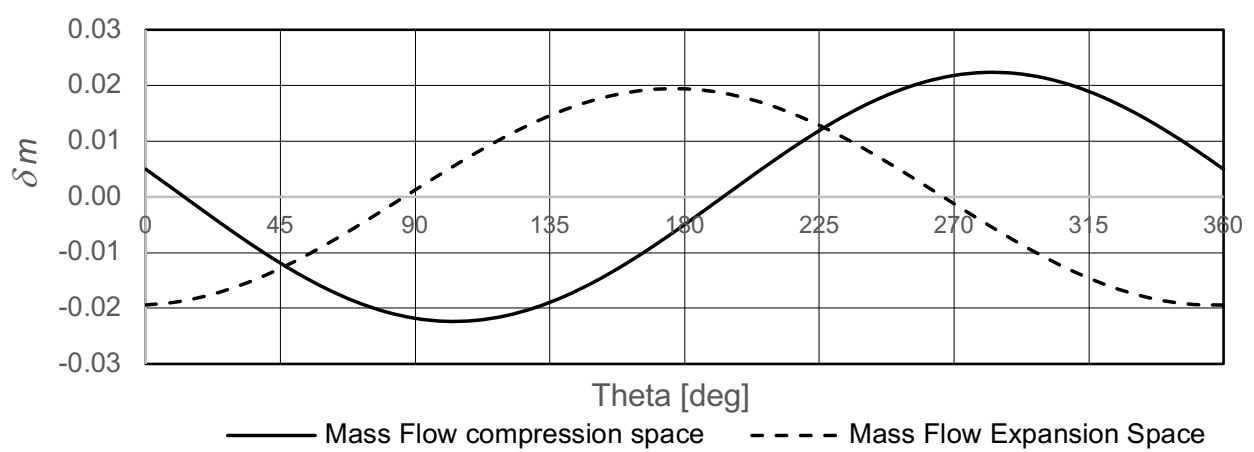

Fig. 8. Working space mass flows - positive into cavity (from Equation A.20)

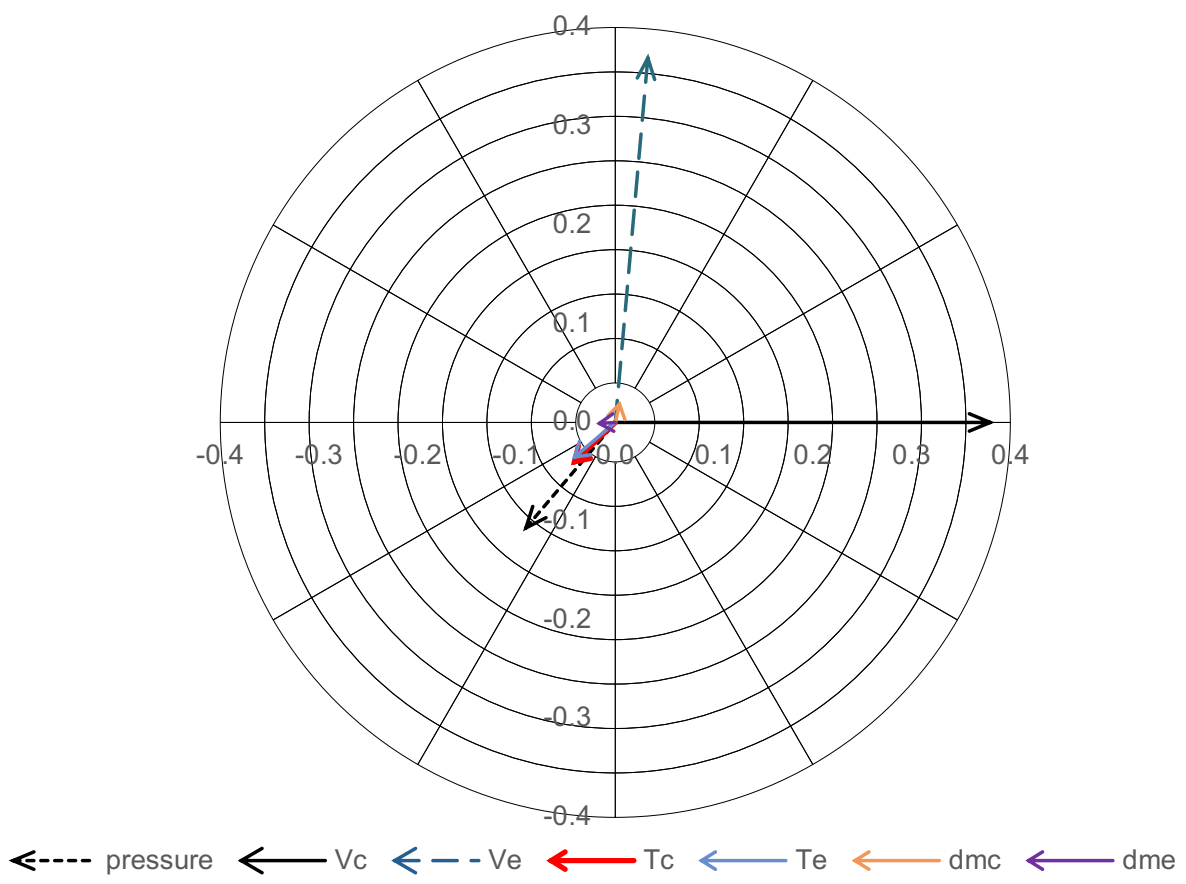

Fig. 9. Phasors relative to $V_{c}$ - Mass Flow Positive into Cavity 


\section{Ideal Efficiency and COP}

Irreversibilities introduced by the adiabatic processes cause the performance of the cycle to be less than Carnot.

$\eta_{a d}=\frac{W_{e}+W_{c}}{Q_{a}}$

Substituting (15) and (16) into (17) gives:

$\eta_{a d}=1+\frac{\left\langle V_{c}\right\rangle \delta V_{c} \sin (\beta)}{\left\langle V_{e}\right\rangle \delta V_{e} \sin (\beta-\alpha)}=1+\frac{1}{\kappa} \frac{\sin (\beta)}{\sin (\beta-\alpha)} \quad$ where $\quad \kappa=\frac{\left|V_{e}\right|}{\left|V_{c}\right|}$

Typically, the phases of the working space temperatures are very close to the pressure phase. Making this assumption on (7), $\delta \hat{p}$ may be approximated.

$\delta p \angle \beta \approx \frac{1}{S}\left[\frac{\left\langle V_{c}\right\rangle}{\left\langle T_{c}\right\rangle}\left(\delta T_{c} \angle \beta-\delta V_{c} \angle 0\right)+\frac{\left\langle V_{e}\right\rangle}{\left\langle T_{e}\right\rangle}\left(\delta T_{e} \angle \beta-\delta V_{e} \angle \alpha\right)\right]$

Solving for $\beta$ and substituting into (18), gives the following rather simple result:

$\eta_{a d} \approx 1-\frac{\left\langle T_{c}\right\rangle}{\left\langle T_{e}\right\rangle}$

Showing that the ideal adiabatic efficiency is only a function of the mean working space temperatures.

Substituting for the $\langle T\rangle_{\mathrm{S}}$ from Equation (A.23) and ignoring second order small terms, the adiabatic efficiency may be represented by the following machine parameters:

$\eta_{a d} \approx 1-\frac{1}{\tau}+\frac{\pi}{2}\left(\frac{\gamma-1}{\gamma}\right)\left(\frac{1}{\tau}+\frac{1}{\kappa}\right) \delta p_{1} \sin \beta \quad$ where $\quad \tau \equiv T_{a} / T_{k}$

This result shows directly that the lower the pressure ratio the closer the efficiency will approach Carnot. Since the adiabatic temperature swings are a direct consequence of the pressure swing, this conclusion may well have been expected. Fig. 10 shows the ideal adiabatic efficiency versus pressure amplitude ratio for typical power engine parameters. The air charged machine has higher ideal performance because the adiabatic temperatures deviate less from the source and sink temperatures.

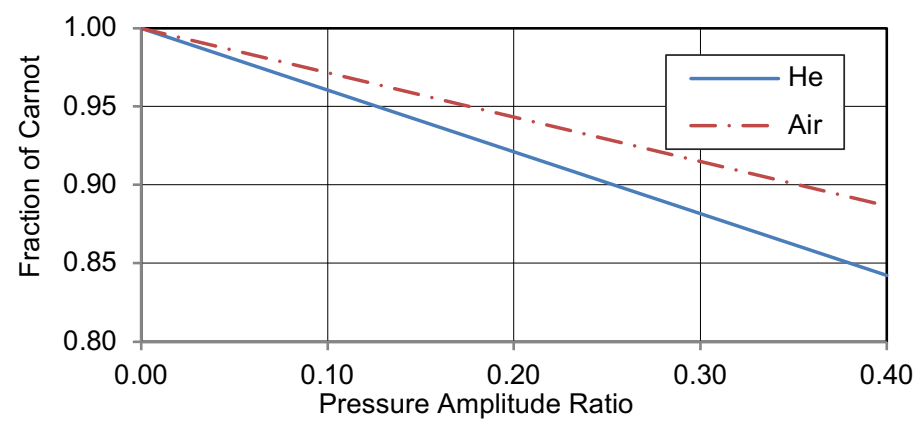

Fig. 10. Efficiency as a fraction of Carnot vs. pressure ratio $(\tau=3.0)$

For cooling engines, the following results are found similarly. 


$$
\begin{aligned}
& \mathrm{COP}_{a d}=\left[1+\frac{\sin \beta}{\kappa \sin (\beta-\alpha)}\right]^{-1} \\
& \mathrm{COP}_{a d}=\frac{\left\langle T_{e}\right\rangle}{\left\langle T_{c}\right\rangle-\left\langle T_{e}\right\rangle} \\
& \mathrm{COP}_{a d} \approx\left[\frac{1}{\tau}-1-\frac{\pi}{2}\left(\frac{\gamma-1}{\gamma}\right)\left(\frac{1}{\tau}+\frac{1}{\kappa}\right) \delta p_{1} \sin \beta\right]^{-1}
\end{aligned}
$$

Since heat pumps operate over a large temperature range it is instructive to see the effect of both temperature and pressure ratios on available COP. Fig. 11 shows the fraction of Carnot versus temperature and pressure ratio for typical machine parameters. For near ambient cooling, the effect of pressure ratio is particularly important.

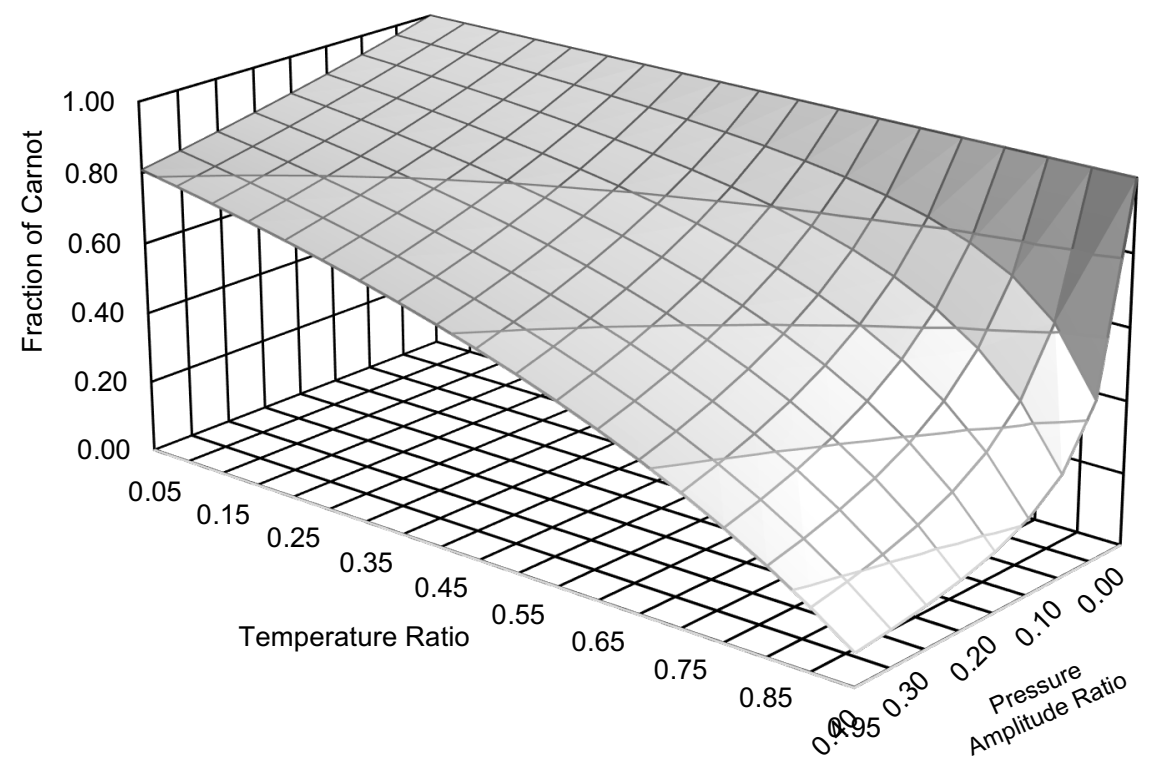

Fig. 11. COP as a fraction of Carnot vs. temperature and pressure amplitude ratios $(\gamma=1.66)$

\section{Ideal Regenerator Heat Flow}

The ideal regenerator heat flow for the Schmidt analysis was originally developed by Creswick [6]. From the First Law, it can be shown that the rate of heat transfer to the regenerator gas is equal to the change in internal energy minus the net enthalpy convected into the gas.

$\mathrm{d} Q_{r}=V_{r} c_{v} \mathrm{~d} p / R-c_{p}\left(T_{k} w_{k r}-T_{a} w_{r a}\right)$

where $w_{k r}$ and $w_{r a}$ are respectively the mass flows across the rejector/regenerator and regenerator/acceptor interfaces. By applying mass balances over the heat exchanger and associated working space, we have:

$w_{k r}=-\mathrm{d} m_{c}-\mathrm{d} m_{k}$ 
$w_{r a}=-\mathrm{d} m_{e}-\mathrm{d} m_{a}$

Since the adiabatic working space temperature variations are typically small with respect to their mean value, we will approximate the ideal gas law from (A.6) as follows:

$\mathrm{d} m=\frac{1}{R\langle T\rangle}(p \mathrm{~d} V+V \mathrm{~d} p) \quad$ where $\langle T\rangle$ is the mean gas temperature.

Substituting (28) into the interface mass flows, (25) becomes:

$\mathrm{d} Q_{r}=\mathrm{j} \frac{\gamma}{\gamma-1}\langle p\rangle\left\{\left[\left(\frac{V_{r}}{\gamma}+V_{k}+V_{a}+\frac{\left\langle V_{c}\right\rangle}{\tau_{c}}+\frac{\left\langle V_{e}\right\rangle}{\tau_{e}}\right) \delta \hat{p}_{1}+\frac{\left\langle V_{c}\right\rangle}{\tau_{c}} \delta \widehat{V}_{c}+\frac{\left\langle V_{e}\right\rangle}{\tau_{e}} \delta \widehat{V}_{e}\right] \mathrm{e}^{\mathrm{j} \theta}+2\left(\frac{\left\langle V_{c}\right\rangle}{\tau_{c}} \delta \widehat{V}_{c}+\right.\right.$

$\left.\left.\frac{\left\langle V_{e}\right\rangle}{\tau_{e}} \delta \hat{V}_{e}\right) \delta \hat{p}_{1} \mathrm{e}^{\mathrm{j} 2 \theta}\right\} \quad$ where $\tau_{c}=\left\langle T_{c}\right\rangle / T_{k}$ and $\tau_{e}=\left\langle T_{e}\right\rangle / T_{a}$

Since the second harmonics do not contribute to the net uni-directional regenerator heat flow:

$\left|Q_{r}\right|=\int_{0}^{\pi} \mathrm{d} Q_{r}=2 \frac{\gamma}{\gamma-1}\langle p\rangle\left|V_{c}\right|\left|\vartheta_{a f} \delta \hat{p}_{1}+\frac{1}{\tau_{c}}+\frac{\kappa}{\tau_{e}} \angle \alpha\right|$

where $\vartheta_{a f}=\frac{\left(V_{r} / \gamma+V_{k}+V_{a}+\left\langle V_{c}\right\rangle / \tau_{c}+\left\langle V_{e}\right\rangle / \tau_{e}\right)}{\left|V_{c}\right|}$ and $\kappa=\frac{\left|V_{e}\right|}{\left|V_{c}\right|}$

The heat transfer ratio is defined.

$\frac{\left|Q_{r}\right|_{i}}{Q_{a i}} \equiv-\frac{2}{\pi} \frac{\gamma}{\gamma-1} \frac{\left|\vartheta_{a f} \delta \hat{p}_{1}+\left(1 / \tau_{c}\right)+\left(\kappa / \tau_{e}\right) \angle \alpha\right|}{\kappa \delta p_{1} \sin (\alpha-\beta)}$

The regenerator effectiveness is given by [7]:

$\varepsilon \equiv \frac{\left|Q_{r}\right|_{\text {act }}}{\left|Q_{r}\right|_{i}}$

where $i$ is the ideal and act is the actual unidirectional heat flow.

For a machine having a non-ideal regenerator, the working gas exits the expansion-side of the regenerator at a different temperature than the acceptor temperature. Thus, energy needs to be transferred at the acceptor to maintain its temperature. The acceptor heat transfer is therefore

$Q_{a}=Q_{a i}+\left|Q_{r}\right|_{i}(1-\varepsilon) \quad$ where $Q_{a i}$ is the ideal acceptor heat transfer.

The indicated efficiency and COP with regerator effectiveness are now

$\eta=\frac{\eta_{a d}}{1+\frac{\left|Q_{r}\right|_{i}}{Q_{a i}}(1-\varepsilon)}$

$\mathrm{COP}=\mathrm{COP}_{a d}\left[1-\frac{\left|Q_{r}\right|_{i}}{Q_{a i}}(1-\varepsilon)\right]$

It is interesting to note is that (31) shows that increasing the pressure amplitude ratio will reduce the heat transfer ratio which reveals a contradiction for the cycle. Referring to the adiabatic cycle efficiency (21) or the COP (24), energy performance is optimized by reducing the $\delta p$ term while improving regenerator performance requires just the opposite. This implies 
an optimum pressure amplitude ratio which is clearly obvious in Figs. 12 and 13. The isothermal (Schmidt) results, compared here, do not show this optimum. This trend has been identified before by simple cycle analysis $[5,8]$.
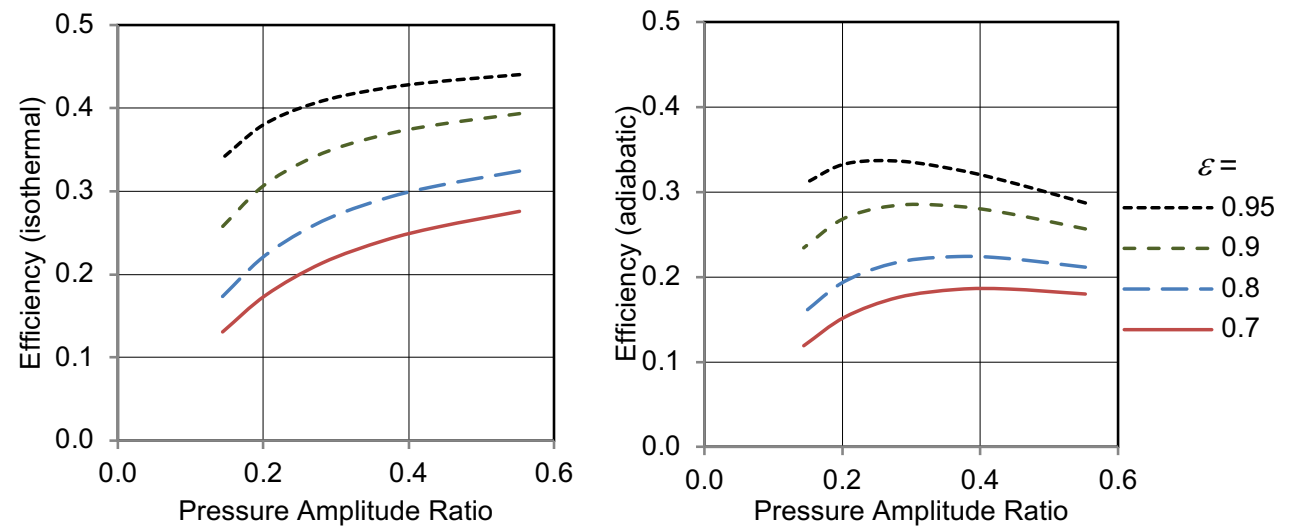

Fig. 12. Efficiency for Isothermal and Adiabatic Power Engine versus $\boldsymbol{\delta} \boldsymbol{p}(\tau=2)$
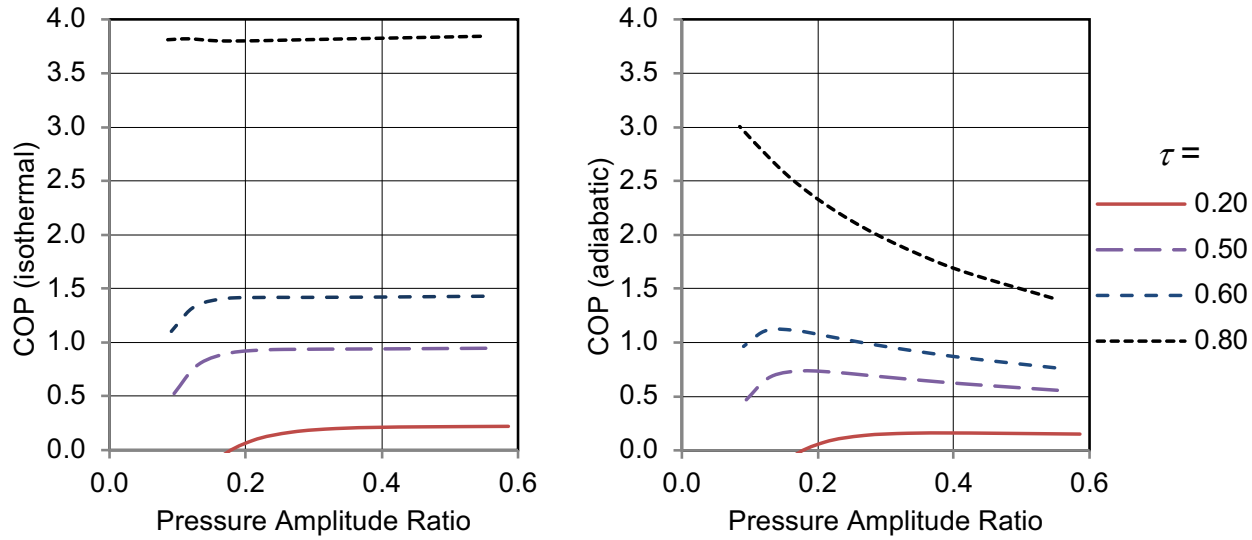

Fig. 13. COP for Isothermal and Adiabatic Cooling Engine versus $\boldsymbol{\delta} \boldsymbol{p}(\varepsilon=0.99)$

Fig. 14 shows the engine efficiency in Fig. 10 with regenerator effectiveness of 0.95 . This completely changes the character of the curves. No longer is the efficiency optimized for lower pressure ratios. Also interesting is that higher pressure amplitude ratios improve aircharged engines more significantly than helium-charged engines. The effect of regenerator effectiveness on cooling engine performance is shown in Fig. 15. In this case he regenerator effectiveness is taken at 0.99. The consequence is quite dramatic when compared to Fig. 11.

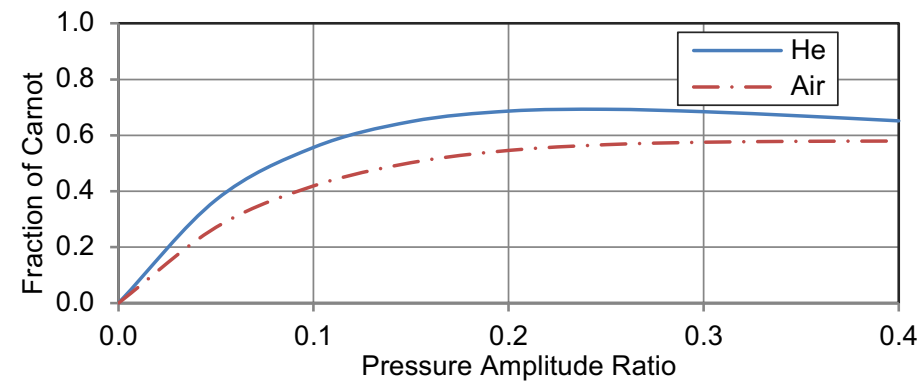

$\varepsilon=0.95$

Fig. 14. Efficiency as a fraction of Carnot with non-ideal regeneration $(\tau=3.0)$ 


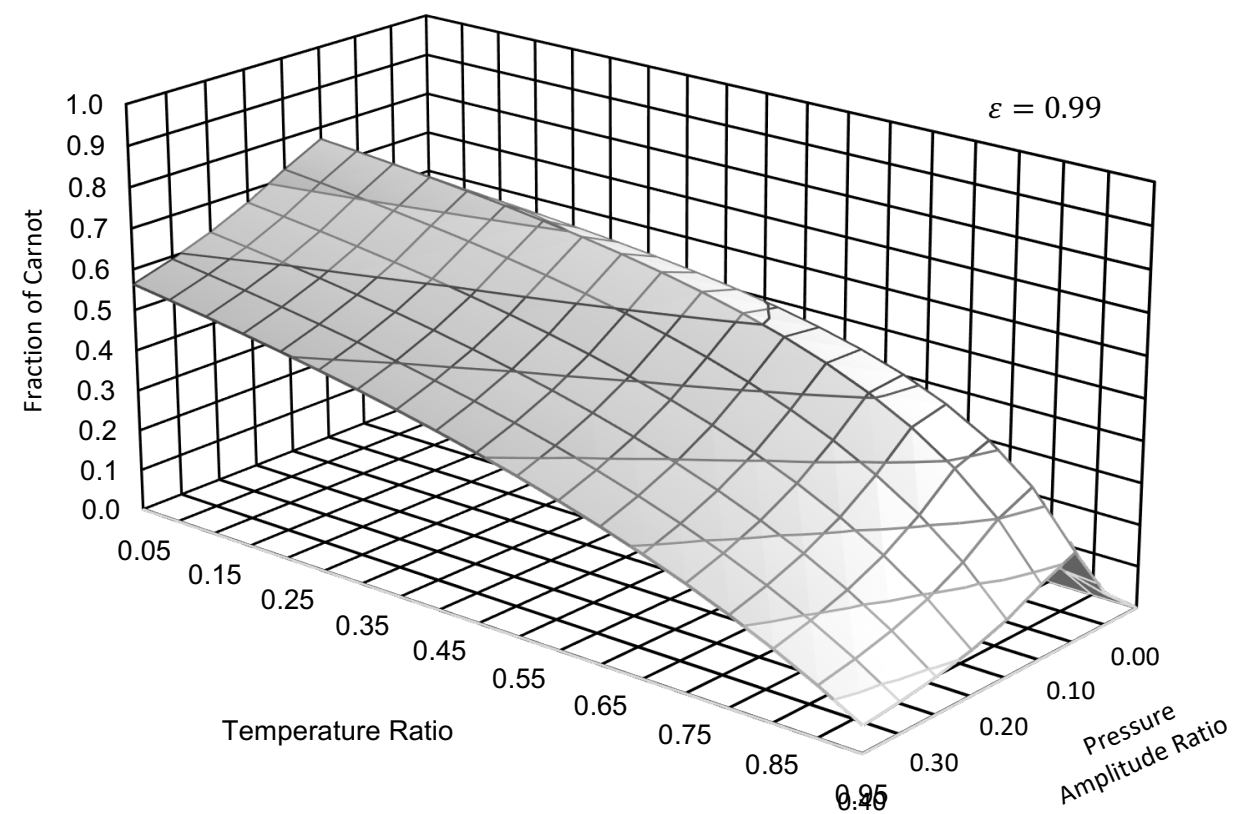

Fig. 15. COP as a fraction of Carnot with non-ideal regeneration $(\gamma=1.66)$

\section{Optimization}

Walker [2, 4] showed that it is possible to non-dimensionalize the Schmidt and adiabatic results by defining three independent variable groups. The groups here are slightly different to those used by Walker owing to the inclusion of the cooler and heater volumes. Also, the temperature ratio used here is the inverse of Walker's. The independent variable groups are therefore:

$$
\begin{array}{ll}
\tau=T_{a} / T_{k} & \text { temperature ratio } \\
\kappa=\left|V_{e}\right| /\left|V_{c}\right| & \text { swept volume ratio } \\
\rho=V_{D} /\left(2\left|V_{c}\right|\right) & \text { dead volume ratio }
\end{array}
$$

where

$$
V_{D}=V_{k}(\tau-1) / \ln \tau+V_{r}+V_{a}(1-1 / \tau) / \ln \tau
$$

and is referred to as the reduced dead volume where for the purposes of optimization, the working space clearance volumes are taken as zero. Therefore each $\delta V=1$.

The following non-dimensional parameters result:

$$
\begin{array}{ll}
p^{\#}=\frac{p}{p_{\max }}=\frac{1-\delta p}{1-\delta p \cos (\beta+\theta)} & \text { pressure variation } \\
Q_{k}^{\#}=\frac{Q_{c}}{V_{t} p_{\max }}=-\frac{\delta \sin \beta}{1+\kappa} & \text { reject heat }
\end{array}
$$


$Q_{a}^{\#}=\frac{Q_{e}}{V_{t} p_{\max }}=\frac{\kappa}{1+\kappa} \delta \sin (\alpha-\beta) \quad$ acceptor heat or lift

$W^{\#}=\frac{W}{V_{t} p_{\max }}=\frac{\delta}{1+\kappa}(\kappa \sin (\alpha-\beta)-\sin \beta) \quad$ work

where $p_{\max }, V_{t}, \delta, \delta p$ and $\beta$ are as follows:

$p_{\max }=\langle p\rangle \frac{\sqrt{1-\delta p^{2}}}{1-\delta p}$

maximum cyclic pressure

$V_{t}=2\left(\left|V_{c}\right|+\left|V_{e}\right|\right)$

sum of the swept volumes

$\delta=\pi\left(\frac{1}{\delta p}-1\right)\left(1-\frac{1}{\sqrt{1-\delta p^{2}}}\right)$

a negative term

$\delta p$ and $\beta$ are obtained from

$\delta \hat{p}=\frac{1}{S^{\#}}\left[\frac{1}{\tau_{c}}\left(\delta \widehat{T}_{c}-1\right)+\frac{\kappa}{\tau} \frac{1}{\tau_{e}}\left(\delta \widehat{T}_{e}-1 \angle \alpha\right)\right]$

where $S^{\#}=\frac{1}{\tau_{c}}+\frac{\kappa}{\tau} \frac{1}{\tau_{e}}+2 \rho \frac{\ln \tau}{\tau-1}, \quad \tau_{c}=\left\langle T_{c}\right\rangle / T_{k} \quad$ and $\quad \tau_{e}=\left\langle T_{e}\right\rangle / T_{a}$

The $\delta T \mathrm{~s}$ and $\langle T\rangle_{\mathrm{s}}$ are obtained as in Appendix A.

$W^{\#}$ indicates the cyclic work for a particular engine size and maximum working gas pressure. The maximum working gas pressure gives an indication of the mass of the engine since it determines the thickness of the pressure vessel walls. Consequently, optimizing $W^{\#}$ for $\kappa$ and $\alpha$ given $\rho$ and $\tau$ would give the highest cylic work for working gas volume and engine mass. Fig.14 and 15 show the isothermal and adiabatic results respectively. Regeneration is taken as perfect and for the adiabatic case, helium is assumed. The optimisation is not accurate for the adiabatic case when $\delta T$ and $\delta p$ begin to approach unity. The isothermal results are not affected by this because the equations are exact. Also, the assumption of zero clearance spaces will introduce error for the adiabatic results due to the linearization (A.17) which again, does not apply to the isothermal result.

\section{Conclusions and Observations}

Ideal analyses should provide useful insights for design. In this regard, the phasor analysis indicates:

1. For adiabatic working spaces, there is a clear optimum pressure amplitude ratio where efficiency or COP is optimized, and this depends strongly on regenerator effectiveness.

2. The pressure amplitude ratio is a fundamental property of the cycle and includes the effects of dead volume. Depending on the location on the thermal performance curve, increasing dead volume may reduce or improve thermal performance insofar as it alters the pressure amplitude ratio (Figs. 12 and 13). This has been noted by Gschwendtner and Bell [14].

3. The ideal adiabatic efficiency or COP depends, in the first order, only on the mean working spaces temperatures.

4. Optimum work or lift for a given maximum pressure and volume requires somewhat different stroke ratios and volume phases for adiabatic and isothermal conditions. 

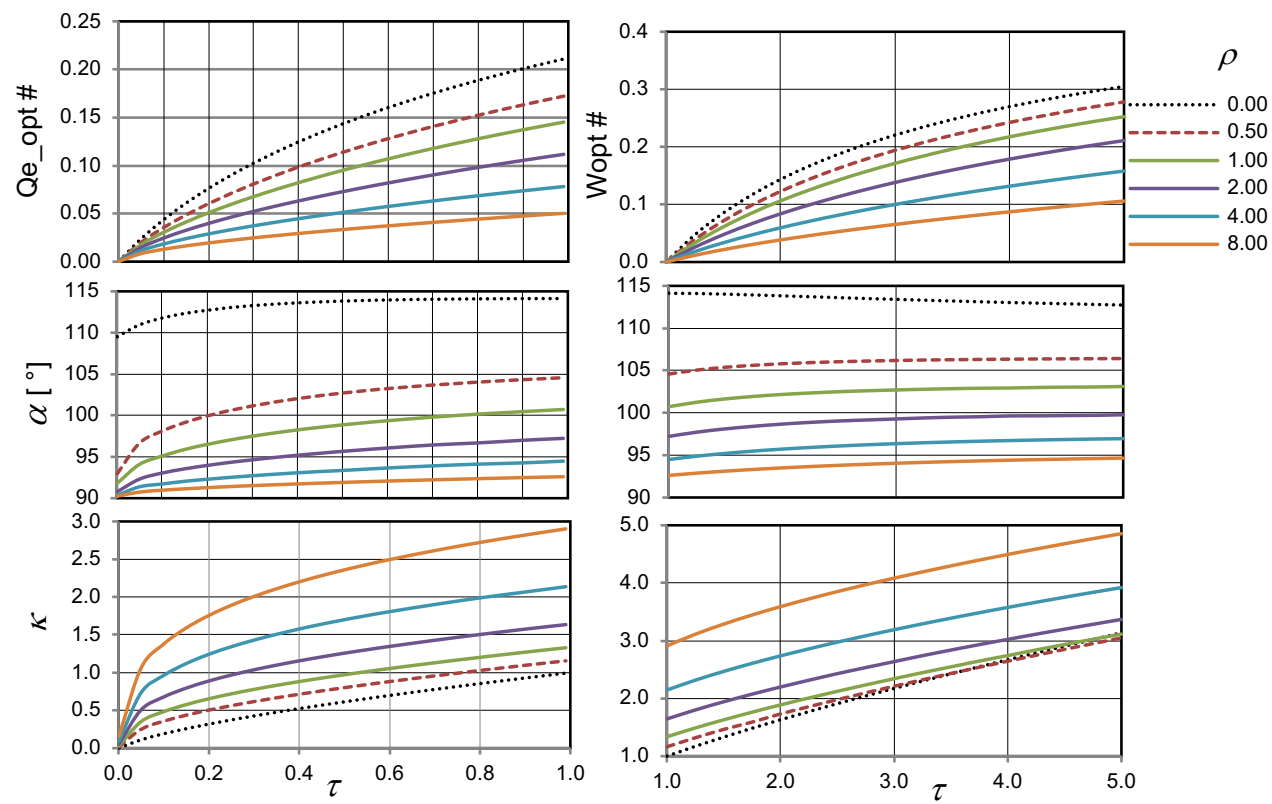

Fig. 14. Isothermal (Schmidt) Optimum Parameters
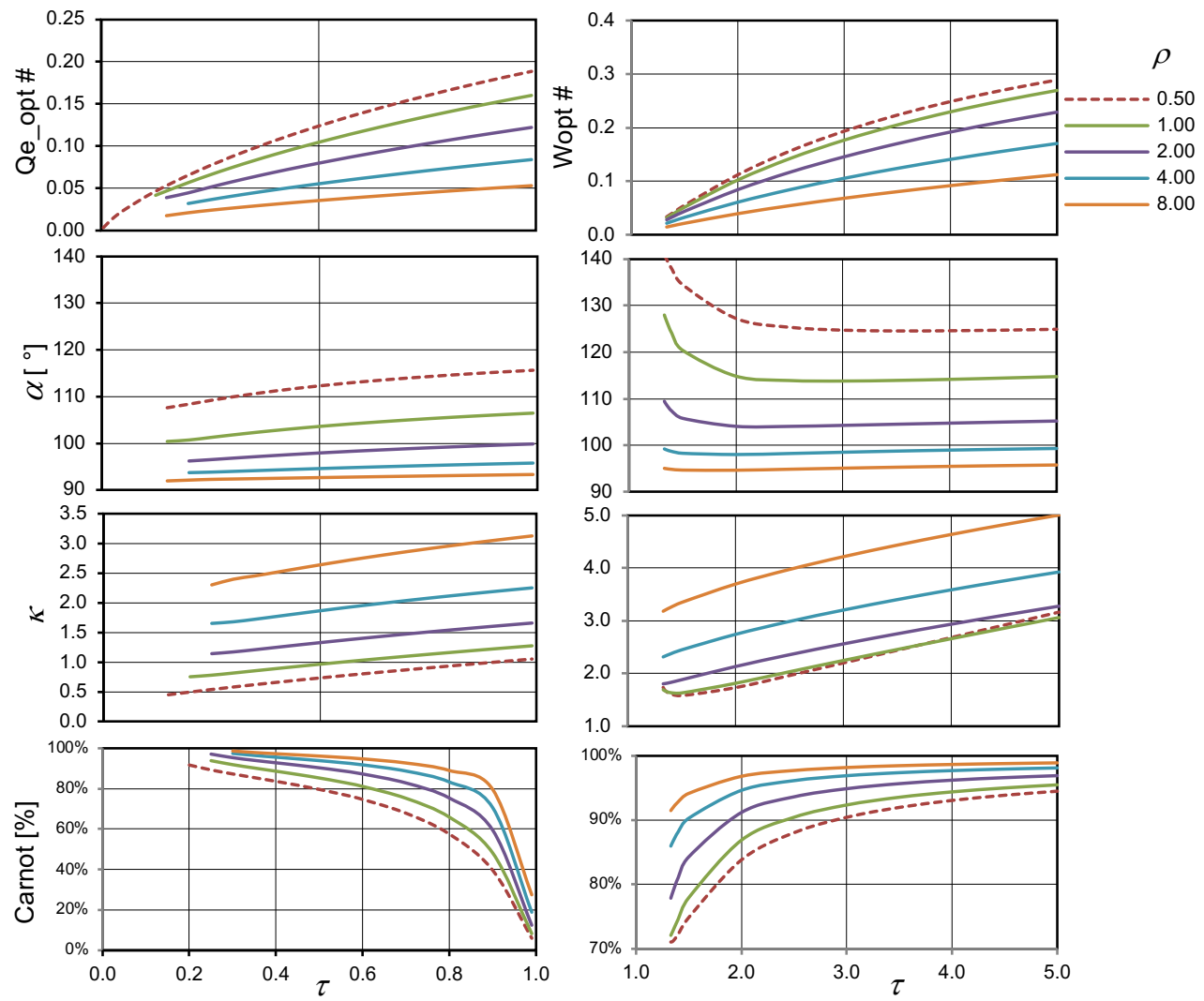

Fig. 15. Adiabatic Optimum Parameters $(\gamma=1.667)$ 
5. And, finally, the phasor analysis offers a generic approach to the condition of the working spaces in that the isothermal result is a simple consequence of the analysis.

Of course, correlation with actual engines will depend on the degree that irreversibilities and losses have been minimized. Table 1 lists some engines to which the analyses have been applied. Regenerator effectiveness and displacer drive power have been accounted for in each case. Calibrated results are taken from a much more extensive simulation that includes parasitic losses and that has been calibrated against actual data. The regenerator effectiveness numbers comes from the simulation and is based on a calculation of the thermal performance of the regenerator. All calculations are based on gas temperatures.

The pressure amplitude ratio and phase tend to be better predicted by the adiabatic analysis versus isothermal. This is important for free-piston dymanics calculations. Energy transfers are quite reasonable even for the Schmidt analysis.

Table 1. Results for Various Machines

\begin{tabular}{|c|c|c|c|c|c|c|c|c|c|}
\hline Machine & Analysis & $\delta p$ & $\begin{array}{c}\delta p \\
\text { phase } \\
{\left[{ }^{\circ}\right]}\end{array}$ & $\varepsilon$ & $\begin{array}{c}\text { Displacer } \\
\text { Power } \\
{[W]}\end{array}$ & $\begin{array}{c}\text { Piston } \\
\text { Power } \\
\text { [W] }\end{array}$ & $\begin{array}{c}\text { Lift } \\
\text { or Heat In } \\
\text { with } \varepsilon[W]\end{array}$ & $\begin{array}{c}\text { Efficiency } \\
\text { or COP } \\
\text { with } \varepsilon\end{array}$ & $\begin{array}{c}\text { Carnot } \\
\text { [\%] }\end{array}$ \\
\hline \multirow{3}{*}{$\begin{array}{l}R E-1000 \\
\text { (power) } \\
{[10]}\end{array}$} & Schmidt & 0.111 & -30.75 & \multirow{3}{*}{0.967} & 171 & 1,109 & 2,619 & 42.3 & 64.7 \\
\hline & Adiabatic & 0.154 & -29.15 & & 234 & 1,463 & 3,382 & 43.3 & 66.2 \\
\hline & Calibrated & 0.146 & -21.30 & & - & 1,019 & 3,553 & 28.7 & 43.9 \\
\hline \multirow{3}{*}{$\begin{array}{l}\text { SPIKE } \\
\text { (power) } \\
\text { [11] }\end{array}$} & Schmidt & 0.115 & -33.46 & \multirow{3}{*}{0.992} & 464 & 2,255 & 4,619 & 48.8 & 71.2 \\
\hline & Adiabatic & 0.132 & -30.96 & & 516 & 2,409 & 5,070 & 47.5 & 69.3 \\
\hline & Calibrated & 0.128 & -21.40 & & - & 1,571 & 5,578 & 28.2 & 41.2 \\
\hline \multirow{3}{*}{$\begin{array}{l}\text { M150 } \\
\text { (cooling) }\end{array}$} & Schmidt & 0.107 & 21.85 & \multirow{3}{*}{0.978} & 4.9 & -101 & \multirow{3}{*}{$\begin{array}{l}78 \text { at }-100^{\circ} \mathrm{C} \\
102 \text { at }-100^{\circ} \mathrm{C} \\
59 \text { at }-100^{\circ} \mathrm{C}\end{array}$} & 0.772 & 64.7 \\
\hline & Adiabatic & 0.133 & 23.16 & & 5.9 & -132 & & 0.773 & 64.8 \\
\hline & Calibrated & 0.137 & 30.00 & & - & -174 & & 0.340 & 28.5 \\
\hline \multirow{3}{*}{$\begin{array}{l}\text { M600 } \\
\text { (cooling) }\end{array}$} & Schmidt & 0.118 & 18.64 & \multirow{3}{*}{0.983} & 21.3 & -407 & \multirow{3}{*}{$\begin{array}{c}320 \text { at }-101^{\circ} \mathrm{C} \\
393 \text { at }-101^{\circ} \mathrm{C} \\
275 \text { at }-101^{\circ} \mathrm{C}\end{array}$} & 0.787 & 71.4 \\
\hline & Adiabatic & 0.143 & 19.57 & & 25.2 & -516 & & 0.762 & 69.1 \\
\hline & Calibrated & 0.147 & 25.80 & & - & -695 & & 0.396 & 35.9 \\
\hline
\end{tabular}

\section{Nomenclature}

Only definitions of parameters not defined in the text are given here.

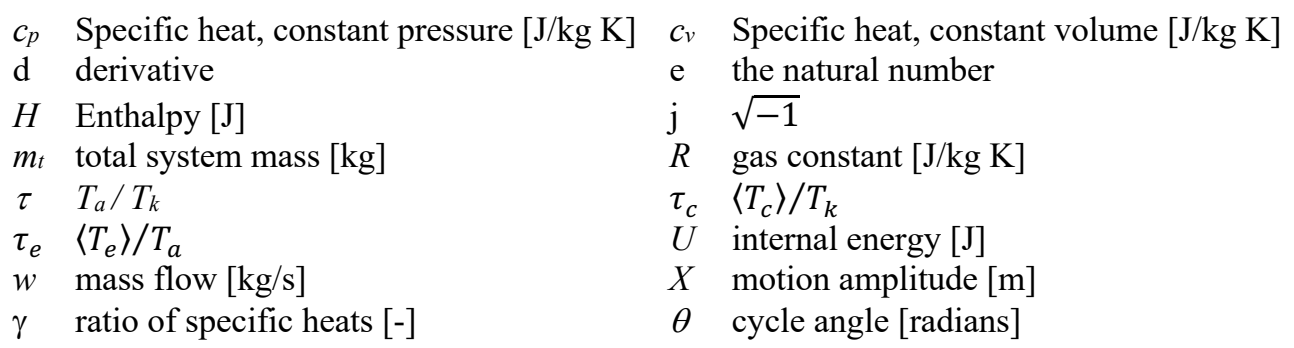

\section{Subscripts}

$a$ acceptor

$r$ regenerator $k \quad$ rejector

1, 2 harmonic number 


\section{Appendix A Working Space Temperatures}

The following is generic for either working space. Since the compression space is the reference phase, $\alpha$ would be taken as zero for that space.

Referring to Fig. A.1, the energy equation for a general adiabatic working space is

$\dot{H}-p \mathrm{~d} V=\mathrm{d} U$

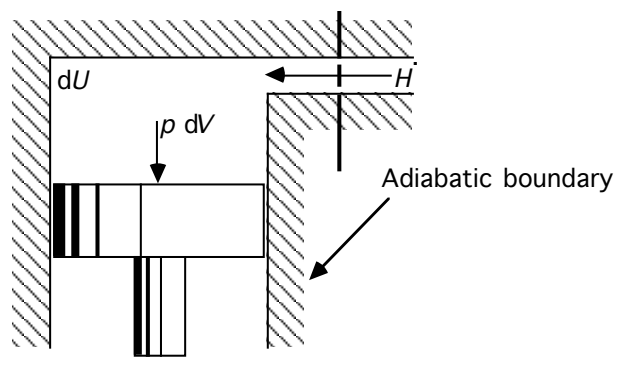

Fig. A.1 The Adiabatic Working Space

From the definition of enthalpy:

$\dot{H}=c_{p} T^{\prime} w$

where $T^{\prime}$ is the temperature of the gas crossing the control volume boundary.

From continuity, the mass flow rate is equal to the rate of change of mass in the working space:

$w=\mathrm{d} m$

(A.2) therefore becomes:

$\dot{H}=c_{p} T^{\prime} \mathrm{d} m$

for $\quad T^{\prime} \leftarrow T$ for $\mathrm{d} m \leq 0$ where $T$ is the working space gas temperature.

$T^{\prime} \leftarrow \bar{T}$ for $\mathrm{d} m>0$ where $\bar{T}$ is the appropriate heat exchanger temperature.

The internal energy is given by $U=c_{v} T m$, thus

$\mathrm{d} U=c_{v}(m \mathrm{~d} T+T \mathrm{~d} m)$

From the gas law and differentiating w.r.t. time:

$\mathrm{d} m=\frac{1}{R}\left(\frac{p}{T} \mathrm{~d} V+\frac{V}{T} \mathrm{~d} p-\frac{p V}{T^{2}} \mathrm{~d} T\right)$

Substituting (A.6), (A.5) and (A.4) into (A.1) noting that $c_{p}=\gamma R /(\gamma-1)$ and $c_{p}-c_{v}=R$, yields the following after simplification: 
$\frac{\mathrm{d} T}{T}=\left(1-\frac{T}{T^{\prime}}\right) \frac{\mathrm{d} V}{V}+\left(1-\frac{T}{\gamma T^{\prime}}\right) \frac{\mathrm{d} p}{p}$

When the flow is out of the working space, ie, $\mathrm{d} m \leq 0$ and $T^{\prime} \leftarrow T$, and (A.7) becomes:

$\frac{\mathrm{d} T}{T}=\left(1-\frac{1}{\gamma}\right) \frac{\mathrm{d} p}{p} \quad$ for $\mathrm{d} m \leq 0$

which is easily integrated to yield

$T=C_{1} p^{(1-1 / \gamma)} \quad$ for $\mathrm{d} m \leq 0$

For gas flow into the working space, ie, $\mathrm{d} m>0$ and $T^{\prime} \leftarrow \bar{T}$, (A.7) becomes:

$\frac{\mathrm{d} T}{T}=\left(1-\frac{T}{\bar{T}}\right) \frac{\mathrm{d} V}{V}+\left(1-\frac{T}{\gamma \bar{T}}\right) \frac{\mathrm{d} p}{p} \quad$ for $\mathrm{d} m>0$

where $\bar{T}$ is the relevant constant heat exchanger temperature.

From (2), noting that $\delta T<1$, (A.10) may be simplified by dropping second order small terms:

$\frac{\mathrm{d} T}{T} \approx\left(1-\frac{\langle T\rangle}{\bar{T}}\right) \frac{\mathrm{d} V}{V}+\left(1-\frac{\langle T\rangle}{\gamma \bar{T}}\right) \frac{\mathrm{d} p}{p}$

which may be integrated to give

$T=C_{2} V^{(1-\tau)} p^{(1-\tau / \gamma)} \quad$ for $\mathrm{d} m>0$

where $\tau=\langle T\rangle / \bar{T}$ and $C_{2}$ is the constant of integration.

Equations (A.9) and (A.12) define the temperature variations in the working spaces.

Taking the first harmonic pressure variation and noting that $\delta p<1$, (A.9) may be simplified by applying Taylor's expansion theorem around $p=\langle p\rangle$ :

$T \approx C_{1}\left[1+\left(1-\frac{1}{\gamma}\right) \delta \hat{p}_{1} e^{j \theta}-\frac{1}{2 \gamma}\left(1-\frac{1}{\gamma}\right) \delta \hat{p}_{1}^{2} e^{j 2 \theta}+\cdots\right] \quad$ for $\mathrm{d} m \leq 0$

where $C_{1}$ is now a new constant and higher order terms have been neglected.

Rewriting (A.13) for easier manipulation:

$T \approx B_{1}+B_{2} \delta \hat{p}_{1} e^{j \theta}-B_{3} \delta \hat{p}_{1}^{2} e^{j 2 \theta} \quad$ for $\mathrm{dm} \leq 0$

where $\quad B_{1}=C_{1}, B_{2}=B_{1}(1-1 / \gamma)$ and $B_{3}=\frac{1}{2 \gamma} B_{2}$

which is the final form of the working space temperature for the part of the cycle where dm $\leq 0$.

Equation (A.12) is treated a little differently. Rewriting (3.92) as the product of two functions $T(p, V)=C_{2} \mathrm{f}(V) \cdot \mathrm{g}(p) \quad$ for $\mathrm{d} m>0$ 
Both $\mathrm{f}(V)$ and $\mathrm{g}(p)$ are respectively non-linear functions of volume and pressure. An approximate method of linearizing (A.15) is to separately linearize $\mathrm{f}(V)$ and $\mathrm{g}(p)$ and then neglect second order small terms from their product.

Linearization of $\mathrm{g}(p)$ about $\langle p\rangle$ is straightforward and is given by

$$
g(p) \approx\langle p\rangle^{(1-\tau / \gamma)}\left[1+(1-\tau / \gamma) \delta \hat{p}_{1} e^{j \theta}-\frac{\tau}{2 \gamma}(1-\tau / \gamma) \delta \hat{p}_{1}^{2} e^{j 2 \theta}+\cdots\right]
$$

The variations of $V$ are a larger fraction of the mean value. In this case additional terms for $\mathrm{f}(V)$ are required from the Taylor's series. Expanding around the mean volume, $\langle V\rangle$, gives:

$$
\mathrm{f}(V) \approx\langle V\rangle^{1-\tau}\left[1+(1-\tau) \delta \widehat{V} e^{j \theta}-\frac{\tau}{2}(1-\tau) \delta \widehat{V}^{2} e^{j 2 \theta}+\frac{\tau}{6}\left(1-\tau^{2}\right) \delta \widehat{V}^{3} e^{j 3 \theta}+\cdots\right]
$$

From (A.16) and (A.17), (A.12) now becomes

$T(p, V) \approx C_{2}\left[1+B_{4} \delta \widehat{V} e^{j \theta}+B_{6} \delta \hat{p}_{1} e^{j \theta}-B_{5} \delta \widehat{V}^{2} e^{j 2 \theta}+B_{4} B_{6} \delta \hat{V} \delta \hat{p} e^{j 2 \theta}-B_{7} \delta \hat{p}_{1}^{2} e^{j 2 \theta} \ldots\right]$

for $\mathrm{d} m>0$

where $B_{4}=1-\tau, B_{5}=\frac{\tau}{2} B_{4}, B_{6}=1-\tau / \gamma, B_{7}=\frac{\tau}{2 \gamma} B_{6}$ and $B_{8}=\frac{\tau}{6}\left(1-\tau^{2}\right)$

and products of small quantities have been neglected and $C_{2}$ is now a new constant.

Collecting harmonic terms:

$T(p, V) \approx C_{2}\left[1+\left(B_{4} \delta \hat{V}+B_{6} \delta \hat{p}_{1}\right) e^{j \theta}+\left(B_{4} B_{6} \delta \hat{V} \delta \hat{p}_{1}-B_{5} \delta \hat{V}^{2}-B_{7} \delta \hat{p}_{1}^{2}\right) e^{j 2 \theta}+\cdots\right]$

for $\mathrm{d} m>0$

To apply the temperature profiles, $\mathrm{d} m$ will be needed. Using the phasor forms of the volumes, pressure and temperatures, (A.6) becomes:

$$
\begin{aligned}
\mathrm{d} m \approx j \omega & \frac{\langle p\rangle\langle V\rangle}{R\langle T\rangle}\left[\delta \hat{V}+\delta \hat{p}_{1}-\delta \widehat{T}\right] e^{j \theta}=w=j \omega\langle m\rangle \delta m e^{j(\varphi+\theta)}=\omega|m| e^{j(\varphi+\pi / 2+\theta)} \\
\mathrm{d} m & >0 \text { for }-(\pi+\varphi)<\theta \leq(-\varphi) \\
\mathrm{d} m & \leq 0 \text { for }(-\varphi)<\theta \leq(\pi-\varphi)
\end{aligned}
$$

Note that the angle for the $w$ phasor is $\varphi+\pi / 2$.

The temperatures in the working spaces for the entire cycle are required in the form given by (2). Using complex Fourier series, the temperatures may be written:

$T=\sum_{n=-\infty}^{\infty} c_{n} e^{j n \theta}$

where $c_{n}=\frac{1}{2 \pi} \int_{-\pi}^{+\pi} T(\theta) e^{-j n \theta} \mathrm{d} \theta$

Giving $c_{0}$ for the mean value: 
$c_{0}=\frac{\left(C_{2}+B_{1}\right)}{2}+\frac{1}{\pi} \delta p_{1}\left(B_{2}-B_{6} C_{2}\right) j e^{j(\beta-\varphi)}-\frac{1}{\pi} B_{4} C_{2} \delta V j e^{j(\alpha-\varphi)}$

And $c_{1}$ for the first harmonic:

$c_{1}=\frac{1}{\pi}\left(C_{2}-B_{1}\right) j e^{j \varphi}+\frac{1}{2}\left[\left(B_{2}+B_{6} C_{2}\right) \delta p_{1} e^{j \beta}+B_{4} C_{2} \delta V e^{j \alpha}\right]+\frac{C_{2}}{\pi}\left(B_{5} \delta V^{2} j e^{j(2 \alpha-\varphi)}-\right.$

$\left.B_{4} B_{6} \delta V \delta p_{1} j e^{j(\alpha+\beta-\varphi)}+B_{7} \delta p_{1}^{2} j e^{j(2 \beta-\varphi)}\right)$

The temperature may now be written in the form of (2) for all $\mathrm{d} m$ with:

$$
\begin{aligned}
\langle T\rangle & =\mathbf{R}\left(c_{0}\right) \\
& =B_{1}\left[\frac{1}{2}-\frac{1}{\pi} \delta p_{1}\left(1-\frac{1}{\gamma}\right) \sin (\beta-\varphi)\right]+C_{2}\left[\frac{1}{2}+\frac{1}{\pi}\left(B_{6} \delta p_{1} \sin (\beta-\varphi)+B_{4} \delta V \sin (\alpha-\varphi)\right)\right]
\end{aligned}
$$

$\langle T\rangle \delta \widehat{T}=B_{1}\left[\frac{1}{2}\left(1-\frac{1}{\gamma}\right) \delta p_{1} e^{j \beta}-\frac{1}{\pi} j e^{j \varphi}\right]+C_{2}\left[\frac{1}{\pi} j e^{j \varphi}+\frac{1}{2}\left(B_{4} \delta V e^{j \alpha}+B_{6} \delta p_{1} e^{j \beta}\right)+\right.$ $\left.\frac{1}{\pi}\left(B_{5} \delta V^{2} e^{j(2 \alpha)}-B_{4} B_{6} \delta V \delta p_{1} e^{j(\alpha+\beta)}+B_{7} \delta p_{1}^{2} e^{j(2 \beta)}\right) j e^{-j \varphi}\right]$

The working spaces temperatures are now fully defined except for the original constants of integration, $B_{1}$ and $C_{2}$, which must be solved from appropriate boundary conditions.

From the energy equation (A.1), and noting that the internal energy over a cycle is zero:

$\oint \dot{H} \mathrm{~d} t=\oint p \mathrm{~d} V$

Which indicates that the net enthalpy flow into the working space is equal to the net $p V$ work done by the piston.

The enthalpy flow into the working space may be evaluated as follows:

$\oint \dot{H} \mathrm{~d} t=\frac{c_{p}}{\omega}\left(\int_{-\pi-\varphi}^{-\varphi} \bar{T} \mathbf{R}(\mathrm{d} m) \mathrm{d} \theta+\int_{-\varphi}^{\pi-\varphi} \mathbf{R}(T) \mathbf{R}(\mathrm{d} m) \mathrm{d} \theta\right)$

where $T$ is the working gas instantaneous temperature and $\bar{T}$ is the associated constant heat exchanger temperature. The limits of integration occur at the zero flow points.

Substituting for $T$ for $\mathrm{d} m \leq 0$ from (A.14) and for $\mathrm{d} m$ from (A.20), (A.25) may be integrated to yield

$\oint \dot{H} \mathrm{~d} t=c_{p}|m|\left(2 \bar{T}-2 B_{1}-\frac{\pi}{2} B_{2} \delta p_{1} \sin (\varphi-\beta)-\frac{2}{3} B_{3} \delta p_{1}^{2} \cos 2(\varphi-\beta)\right)$

where $|m|=\langle m\rangle \delta m$

The $p V$ work is given by (14), and combined with (10), gives

$W=2 \pi\langle V\rangle\langle p\rangle \frac{\delta V}{\delta p}\left(\sqrt{1-\delta p^{2}}-1\right) \sin (\alpha-\beta)=-\pi\langle V\rangle\langle p\rangle \delta p_{1} \sin (\alpha-\beta)$

Equating (A.26) and (A.27), and noting the definitions of $B_{1}, B_{2}$ and $B_{3}$ from (A.14), the constant $B_{1}$ may be solved for: 
$B_{1}=\left(\bar{T}-\frac{W}{2 c_{p}|m|}\right)\left[1+\frac{\pi}{4}\left(1-\frac{1}{\gamma}\right) \delta p_{1} \sin (\varphi-\beta)+\frac{1}{6 \gamma}\left(1-\frac{1}{\gamma}\right) \delta p_{1}{ }^{2} \cos 2(\varphi-\beta)\right]^{-1}$

For the working space temperatures to be continuous, the temperatures for each direction of flow must be equal at $\theta=-\varphi$ and $\theta=\pi-\varphi$. From (A.9) and (A.12) and taking the average value to account for non-linearity:

$C_{2 \_1}=B_{1}\left[1+\delta p_{1} \cos (\beta-\varphi)\right]^{(\tau-1) / \gamma}[1+\delta V \cos (\alpha-\varphi)]^{(\tau-1)}$ at $\theta=-\varphi$

$C_{2 \_}=B_{1}\left[1-\delta p_{1} \cos (\beta-\varphi)\right]^{(\tau-1) / \gamma}[1-\delta V \cos (\alpha-\varphi)]^{(\tau-1)}$ at $\theta=\pi-\varphi$

$C_{2}=\frac{1}{2}\left(C_{2 \_1}+C_{2 \_}\right) \approx B_{1}\left(1+\frac{(\tau-1)^{2}}{\gamma} \delta p_{1} \delta V \cos (\alpha-\varphi) \cos (\beta-\varphi)\right) \approx B_{1}$

This completes the necessary equations to solve the temperature variations in the working spaces.

\section{References}

1. G. Schmidt, Theory der Lehmann'schen calorischen machine, Zeitschrift des Vereines Deutscher Ingenieure, 15, part 1, pp 1-12, (Jan 1871), part 2, pp 97-112 (Feb 1871)

2. G. Walker, Stirling Cycle Machines (1973)

3. I. Urieli, A Computer Simulation of Stirling Cycle Machines, Ph.D. Thesis, University of the Witwatersrand (1977)

4. G. Walker, Stirling Cycle Engines (1980)

5. G.T. Reader and C. Hooper, Stirling Engines (1983)

6. F.A. Creswick, Thermal design of Stirling cycle machines, SAE Trans, Int. Auto. Eng. Congr., paper 650079 (1965).

7. G.Y. Umarov, T. P. Tursunbaev, Lashkareva and V.S. Trukhov, Influence of regenerator efficiency on the thermal efficiency of a Stirling engine dynamic energy converter, Geliotekhnika, 9, no 3, (Applied Solar Energy - USSR, English translation), pp 58-61 (1973)

8. C.J. Rallis, I. Urieli and D.M. Berchowitz, A new ported constant volume external heat supply regenerative cycle. Proc. $12^{\text {th }}$ IECEC, paper 779256, pp 1534-1537 (Aug 1977)

9. G. Petit Bois, Tables of Indefinite Integrals, Dover (1961)

10. J. Schreiber, Test Results and Description of a $1 \mathrm{~kW}$ Free-Piston Stirling Engine with a Dashpot Load. Proc 18th IECEC, paper 839145, pp 887-896 (Aug 1983)

11. D.M. Berchowitz, The development of a $1 \mathrm{~kW}$ electrical output free piston Stirling engine alternator unit, Proc 18th IECEC, paper 839146, pp 897-901 (Aug 1983)

12. D.M. Berchowitz, A phasor description of the Stirling Cycle, Proc $16^{\text {th }}$ ISEC, (2016)

13. N.C. Chen, F.P. Griffin and C.D. West, Linear Harmonic Analysis of Stirling Engine Thermodynamics, Oak Ridge National Laboratory, (Aug 1984)

14. M. Gschwendtner and G. Bell, The myth about dead volume in Stirling engines, Proc. of the Inst. of Mech, Engineers, Mechanical Engineering Science, 231, issue 19, pp 3665-3675 (Oct 2017) 